\title{
Contribution à l'étude de l'infection à Rhodococcus equi chez les équidés dans le nord-est de la Tunisie
}

\author{
A. Chabchoub ${ }^{1 *}$ S. Taouji ${ }^{2}$ F. Landolsi ${ }^{1}$ \\ C. Collobert ${ }^{2}$ Z. Chtioui ${ }^{1}$ L. Messadi ${ }^{1}$ \\ M. Ezzaouia ${ }^{3}$ A. Ghorbel ${ }^{1}$
}

\begin{abstract}
Mots-clés
Equidae - Cheval - Rhodococcus
\end{abstract}

\section{Résumé}

Les auteurs ont recherché par la technique Elisa la présence d'anticorps antiprotéine de virulence VAP A de Rhodococcus equi chez 39 chevaux ayant présenté des symptômes évoquant la rhodococcose et chez 57 poulains sains considérés comme témoins. Parmi les chevaux malades, 35,9 p. 100 ont été positifs contre 17,5 p. 100 seulement chez les témoins. Aucune différence n'a été relevée en fonction du sexe ni de la race. Néanmoins, les résultats sérologiques ont montré des différences statistiquement significatives entre les tranches d'âges des chevaux de l'étude.

\section{INTRODUCTION}

La pathologie néonatale du poulain a souvent des répercussions graves aussi bien sur le plan médical que sur le plan économique. Plusieurs causes sont à l'origine de cette entité notamment la recrudescence de la pathologie induite par Rhodococcus equi durant ces dernières années. La rhodococcose équine est due à un bacille polymorphe à Gram positif initialement connu sous le nom de Corynebacterium equi et récemment sous le nom de Rhodococcus equi. C'est une maladie qui sévit principalement dans les régions d'élevage chez les poulains. Cette infection a été décrite pour la première fois en 1923 par Magnusson chez de jeunes poulains en Suède. Elle peut être observée chez l'homme surtout chez les individus immunodéprimés ainsi que chez plusieurs autres espèces animales (16). Du fait de son importance, l'infection à Rhodococcus equi a fait l'objet de plusieurs travaux dans le monde. En Tunisie, des observations suspectant la présence de la maladie ont été rapportées par l'un des auteurs, mais aucune étude sur l'impact réel de cette maladie n'a été effectuée.

Le présent travail a consisté à rechercher les anticorps antiprotéine de virulence VAP A de Rhodococcus equi chez des équidés dans le

1. Ecole nationale de médecine vétérinaire, Sidi-Thabet 2020, Tunisie

2. Agence française de sécurité sanitaire des aliments (Afssa), Dozulé, Goustranville 14430, France

3. Fondation nationale d'amélioration de la race chevaline (Fnarc), Sidi-Thabet 2020, Tunisie

* Auteur pour la correspondance

Ecole nationale de médecine vétérinaire, Service de pathologie médicale, Sidi-Thabet 2020, Tunisie ; e-mail : chabchoub.ahmed@iresa.agrinet.tn nord-est de la Tunisie, à évaluer l'impact de l'infection sur l'échantillon équin et à apporter, dans la mesure du possible, quelques informations sur le degré et la manière de son évolution.

La technique Elisa utilisée pour les analyses a été mise au point par le laboratoire d'études et de recherches en pathologie équine (Lerpe, Afssa, Dozulé, France). Cette technique permet la recherche des anticorps antiprotéine de virulence VAP A de Rhodococcus equi.

L'étude a été réalisée sur un échantillon de 96 équidés, essentiellement des poulains de différentes races, répartis sur plusieurs élevages du nord-est de la Tunisie.

\section{MATERIEL ET METHODES}

Le travail a consisté à effectuer une étude clinique associée à des prélèvements sanguins à partir de la veine jugulaire de 39 équidés malades ( 32 poulains et 7 adultes) présentant des symptômes évoquant la présence de Rhodococcus equi et 57 poulains sains utilisés comme témoins. L'ensemble de l'effectif étudié provenait de plusieurs élevages équins. Tous les élevages équins organisés ont été systématiquement visités (haras étatiques ou privés agréés pour la monte publique) dans la région du nord-est (gouvernorats de Tunis, Ariana, Mannouba et Bizerte) et chaque fois que des équidés ont présenté une symptomatologie évoquant la rhodococcose (atteintes respiratoires, digestives et/ou des troubles locomoteurs) (4) un prélèvement a été effectué. Par ailleurs, des prélèvements ont été effectués sur des équidés sains vivant dans les mêmes conditions. 
Rhodococcus equi, isolé du poumon de poulain atteint de pneumonie, a été cultivé dans un bouillon d'infusion de cerveau et de cœur (BHIB) pendant $72 \mathrm{~h}$ à $37{ }^{\circ} \mathrm{C}$ puis agité à 1500 tours par min (150 rpm). Les cellules ont ensuite été récoltées par centrifugation,

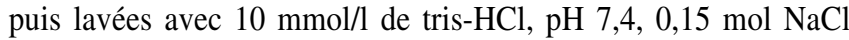
(Tbs buffer) et recentrifugées. Les cellules (30-50 mg poids humide) ont été ajoutées à une solution à 2 p. 100 de triton X 114 dans le Tbs avec $1 \mathrm{mmol} / \mathrm{l}$ de fluorure de phénylméthylsulfonyle comme inhibiteur des protéases puis agitées toute la nuit à $+4{ }^{\circ} \mathrm{C}$. La matière insoluble a été éliminée par centrifugation à $+4^{\circ} \mathrm{C}$ et le surnageant a été récupéré et chauffé à $37^{\circ} \mathrm{C}$ pendant 10 min avant recentrifugation à $25^{\circ} \mathrm{C}$ pendant $15 \mathrm{~min}$. La phase détergente a été lavée une fois avec assez de Tbs pour constituer une solution à 2 p. 100 de TX-114 qui a été mélangée à 10 volumes d'acétone puis laissée toute la nuit à $-20{ }^{\circ} \mathrm{C}$ pour précipitation. Les antigènes VAP A ont alors été isolés et récupérés pour les plaques Elisa selon le protocole de Takai et coll. (17) et de Prescott et coll. (12). Après incubation durant $60 \mathrm{~min}$ à la température ambiante, la densité optique a été mesurée dans un lecteur Elisa automatique à $405 \mathrm{~nm}$. Les puits contenant uniquement l'antigène ont été utilisés comme «blanc ». Le titre Elisa a été exprimé comme la dernière dilution du sérum donnant deux fois l'indication de la densité optique d'un contrôle négatif du sérum dilué au 1/10 (0,045 unité DO).

Les différences entre les groupes ont été établies par un test non paramétrique $\left(\chi^{2}\right)$ pour les pourcentages. La différence a été considérée significative au risque d'erreur de 5 p. 100.

\section{RESULTATS}

\section{Importance des signes cliniques}

Sur les 39 équidés malades, 23 ont présenté une symptomatologie respiratoire (broncho-pneumonie, jetage, toux...), 7 des troubles digestifs (coliques, diarrhées...), 7 des signes ostéoarticulaires et musculaires (tuméfaction des jarrets, arthrite...) et 2 poulains ont présenté des signes cutanés (abcès multiples généralisés). Parmi les animaux malades, sept sont morts, six suite à des symptômes respiratoires et un après des troubles digestifs.

\section{Prévalence de l'infection à Rhodococcus equi}

Sur les 96 équidés examinés pour la recherche d'anticorps contre la protéine de virulence VAP A de Rhodococcus equi, 24 se sont révélés positifs, soit une prévalence globale de 25 p. 100. Parmi les 39 équidés suspects, 14 se sont révélés positifs $(35,9$ p. 100) (tableau I). Parmi les animaux témoins, 10 se sont révélés positifs (17,5 p. 100). La différence de la prévalence de l'infection entre les deux lots a été statistiquement significative $\left(\chi^{2}=4,08\right)$. Sur les 53 mâles examinés, 12 se sont révélés positifs (22,65 p. 100). Parmi les femelles, 12 sur les 43 étudiées ont été positives (27,9 p.100) (tableau II). La différence entre la prévalence de l'infection à Rhodococcus equi en fonction du sexe n'a pas été statistiquement significative $\left(\chi^{2}=0,053\right)$.

\section{Tableau}

Prévalence globale de l'infection à Rhodococcus equi

\begin{tabular}{lclc} 
Test Elisa & Animaux malades & Témoins & Total \\
\cline { 2 - 4 } & Nb. $(\%)$ & Nb. $(\%)$ & Nb. $(\%)$ \\
\hline Positifs & $14(35,90)$ & $10(17,55)$ & $24(25)$ \\
Négatifs & $25(64,10)$ & $47(82,46)$ & $72(75)$ \\
Total & $39(100)$ & $57(100)$ & $96(100)$
\end{tabular}

Tableau II

Prévalence de l'infection à Rhodococcus equi en fonction du sexe

\begin{tabular}{llrr} 
& Test Elisa & Femelles (20) & \multicolumn{1}{c}{ Mâles (19) } \\
\cline { 3 - 4 } & & Nb. $(\%)$ & \multicolumn{1}{c}{ Nb. $(\%)$} \\
Chevaux & Positifs & $7(35)$ & $7(36,84)$ \\
malades & Négatifs & $13(65)$ & $12(63,15)$ \\
Témoins & Positifs & $5(21,7)$ & $5(14,7)$ \\
& Négatifs & $18(78,26)$ & $29(85,29)$ \\
Total & Positifs & $12(27,9)$ & $12(22,65)$ \\
& Négatifs & $31(72)$ & $41(77,35)$
\end{tabular}

L'effectif en fonction de l'âge a été arbitrairement partagé en quatre groupes. Dans le premier groupe, les animaux ont été âgés de 1 jour à 3 mois, dans le deuxième groupe de 3 à 6 mois, dans le troisième groupe de 6 et 12 mois et dans le quatrième groupe de plus d'un an. Dans le premier groupe, deux poulains sur les 17 se sont révélés positifs (11,7 p. 100) (tableau III). Dans le deuxième groupe, 16 parmi les 38 poulains se sont révélés positifs $(42,1$ p. 100). Dans le troisième groupe, deux parmi les 22 chevaux examinés ont été positifs $(9,1$ p. 100). Enfin, dans le quatrième groupe, quatre chevaux sur les 19 examinés ont été positifs (21,1 p. 100). La différence a été statistiquement significative entre la prévalence de l'infection à Rhodococcus equi dans les groupes 1 et $2\left(\chi^{2}=4,89\right)$ ainsi que 2 et $3\left(\chi^{2}=9,84\right)$.

Sur les 65 chevaux pur-sang Arabes, 16 ont été positifs, soit une prévalence de 24,6 p. 100 (tableau IV). Dans le groupe des chevaux pur-sang Anglais testés, quatre parmi les dix examinés se sont révélés positifs (40 p. 100). Dans le groupe des chevaux Barbes et Arabes-Barbes, quatre ont été positifs parmi les 16 chevaux examinés (25 p. 100). Il n'y a pas eu de différence statistiquement significative pour la prévalence de l'infection à Rhodococcus equi en fonction de la race des chevaux examinés $\left(\chi^{2}=3,99\right)$.

Par ailleurs, aucune trace sérologique de Rhodococcus equi n'a été trouvée chez les ânes, les mulets et les poneys examinés.

\section{Tableau III}

Prévalence de l'infection à Rhodococcus equi en fonction de l'âge

\begin{tabular}{|c|c|c|c|c|c|}
\hline \multirow[t]{2}{*}{ Test Elisa } & 1 jour-3 mois & 3 mois- 6 mois & 6 mois-1 an & $>1$ an & Total \\
\hline & Nb. (\%) & Nb. $(\%)$ & Nb. (\%) & Nb. (\%) & Nb. (\%) \\
\hline Positifs & $2(11,76)$ & $16(42,10)$ & $2(9,09)$ & $4(21,05)$ & $24(25)$ \\
\hline Négatifs & $15(88,24)$ & $22(57,79)$ & $20(90,90)$ & $15(78,94)$ & $72(75)$ \\
\hline Total & $17(100)$ & 38 (100) & $22(100)$ & $19(100)$ & $96(100)$ \\
\hline
\end{tabular}




\section{Tableau IV}

Prévalence de l'infection à Rhodococcus en fonction de la race

\begin{tabular}{lccc} 
& $\begin{array}{c}\text { Test Elisa } \\
\text { positif }\end{array}$ & $\begin{array}{c}\text { Test Elisa } \\
\text { négatif }\end{array}$ & Total \\
& Nb. (\%) & Nb. (\%) & Nb. (\%) \\
\hline $\begin{array}{l}\text { Pur-sang Arabes } \\
\text { Pur-sang Anglais }\end{array}$ & $16(24)$ & $49(75)$ & $65(71,4)$ \\
$\begin{array}{l}\text { Barbes et } \\
\text { Arabes Barbes }\end{array}$ & $4(40)$ & $6(60)$ & $10(11)$ \\
Total & $4(25)$ & $12(75)$ & $16(17,6)$ \\
& $24(100)$ & $67(100)$ & $91(100)$
\end{tabular}

\section{DISCUSSION}

Dans cette étude, une série d'examens cliniques et de prélèvements sanguins ont été effectués sur des équidés présentant des signes cliniques évoquant l'infection à Rhodococcus equi et des poulains sains qui ont servi de témoins.

La rhodococcose équine est une maladie qui sévit essentiellement en période chaude $(1,7)$. Pour cette raison, la période des prélèvements a volontairement été limitée d'août à d'octobre. Les auteurs se sont ainsi déplacés dans de nombreux élevages puisque, à cette époque de l'année, les poulains quittent les haras nationaux qui constituent, pour la majorité d'entre eux, leur lieu de naissance.

La technique utilisée a été l'Elisa. Elle est considérée comme la meilleure méthode de diagnostic de l'infection à Rhodococcus equi en raison d'une sensibilité et d'une spécificité supérieures à celles des autres méthodes sérologiques (AGID et IHA) $(7,17)$.

En effet, c'est une technique rapide à réaliser à partir d'une simple prise de sang et dotée d'une bonne valeur prédictive (14). Elle permet au clinicien d'établir un diagnostic précoce de l'infection, dès l'apparition des premiers symptômes, et de confirmer également une infection à Rhodococcus equi (17). Néanmoins, la technique Elisa n'est pas suffisante et une confirmation bactériologique demeure nécessaire (17).

Cette technique possède cependant certaines limites. En effet, la mesure du titre d'anticorps dirigés contre la protéine de virulence VAP A, fortement exprimée par les souches virulentes de Rhodococcus equi, est conditionnée par le choix de la souche dans la préparation de la solution antigénique par l'institut du cheval (Afssa, Dozulé, France). Aussi un titre élevé de ces anticorps anti-VAP A pourrait être détecté chez des animaux en bonne santé (14). De plus, Pronost et coll. estiment que le nombre de réponses faussement positives qui peuvent être données par cette technique est difficile à évaluer à cause du traitement mis en œuvre dès la connaissance du résultat (14).

Les anticorps dirigés contre la protéine de virulence VAP A de Rhodococcus equi ont été mis en évidence par la technique Elisa en quantité importante chez 14 sujets (35,9 p. 100) parmi les 39 chevaux présentant des symptômes évoquant une infection à Rhodococcus equi.

Ainsi, les auteurs peuvent affirmer avec certitude la présence et la circulation de Rhodococcus equi en Tunisie. De plus, cette affirmation concorde avec les travaux de l'un des auteurs qui a isolé cette bactérie chez deux poulains morts suite à une broncho-pneumonie.

La prévalence trouvée a été de 35,9 p. 100. Elle est comparable à la moyenne des prévalences rapportées dans d'autres études $(7,17)$ réalisées grâce à la technique Elisa utilisant la souche ATCC 6939 et portant sur des poulains âgés de 45 jours appartenant à trois catégories d'élevage : des élevages où l'évolution de la maladie est sporadique (19,7 p. 100), des élevages où l'évolution est endémique $(41,7$ p. 100) et des élevages qui n'ont pas connu la maladie $(8,1$ p. 100$)$.

Néanmoins, Collobert et coll. (4) rapportent dans une étude étalée sur dix années et portant sur 863 chevaux à l'autopsie, un taux de 8,69 p. 100 avec des variations annuelles de la prévalence dont le taux le plus élevé (taux de 14) a été observé sur un effectif de 50 chevaux en 1995.

Dans la présente étude, dix poulains parmi les 57 chevaux témoins ont eu un résultat sérologique positif vis-à-vis de l'infection, bien qu'ils n'aient présenté aucun signe de la maladie. Cela pourrait signifier une éventuelle évolution de l'infection sans aucune manifestation symptomatologique (7) ou un degré de virulence variable au sein des souches en circulation (14) ou encore un contact de l'équidé avec l'agent pathogène (7).

A l'opposé, des symptômes respiratoires graves à l'origine même de la mort de l'animal ont été notés, alors que la sérologie avait été négative. Ceci rejoint les résultats de Hofmann et coll. et s'expliquerait par le fait que d'autres agents pathogènes, tels que Streptococcus equi, Streptococcus zooepidemicus, pourraient être à l'origine d'une symptomatologie similaire (8).

L'étude statistique a montré une différence significative entre la prévalence de l'infection à Rhodococcus equi chez les chevaux suspects et celle des poulains témoins, ce qui est en accord avec ce qui a été rapporté par ailleurs $(6,7,17)$.

Il n'y a pas eu de différence significative de la prévalence de l'infection entre les chevaux mâles et femelles, ce qui concorde avec ce qui a été rapporté par Prescott et coll. (13).

Les résultats sérologiques obtenus par tranches d'âges ont montré des différences significatives entre deux des quatre groupes de chevaux, ce qui concorde avec ce qui a été rapporté par Knottenbelt en 1993 (10). En effet, ce dernier mentionne que les poulains âgés de 2 à 4 mois sont les plus atteints, ce qui est lié à une chute physiologique du titre des anticorps maternels à un moment où leur système immunitaire est encore immature. Dans ce cas, l'infection à Rhodococcus equi chez les jeunes poulains peut être qualifiée d'opportuniste (14).

La classe des équidés adultes qui présentaient des symptômes suspects était constituée d'équidés en mauvais état de santé dont quatre étaient morts quelques jours après le prélèvement. L'éventuelle infection des chevaux adultes a été rapportée par Hondalus qui a mentionné l'intervention d'une immunodépression chez ces animaux suite à l'évolution de maladies intercurrentes (9).

L'absence de différence significative de la prévalence de l'infection à Rhodococcus equi entre diverses races (pur-sang Arabe, pur-sang Anglais, Barbe et Arabe-Barbe) concorde avec ce qui a été rapporté par Pronost et coll. (14). En effet, ils mentionnent que toutes les races peuvent être infectées de manière équivalente par Rhodococcus equi. Néanmoins, les résultats présentés ici sont en désaccord avec ceux de Falcon et coll. qui observent que les chevaux pur-sang Arabes sont plus atteints que les autres races (5).

Les ânes, les poneys et les mulets examinés ont tous été négatifs. Etant donné leur faible nombre, on ne peut cependant pas tirer de conclusion sur leur sensibilité à l'infection à Rhodococcus equi. A la connaissance des auteurs, aucune étude antérieure n'a évoqué l'infection de l'âne et du mulet par Rhodococcus equi. Néanmoins, Martens et coll. en 1982 (11) ont utilisé des poneys dans une étude expérimentale sur l'infection à Rhodococcus equi. 


\section{CONCLUSION}

L'importance de l'infection à Rhodococcus equi chez les chevaux dans le monde a amené les auteurs à étudier son impact en Tunisie. Une enquête clinique et sérologique a été menée sur 96 équidés répartis sur plusieurs élevages. L'existence d'un titre élevé d'anticorps contre Rhodococcus equi a été mise en évidence, pour la première fois en Tunisie par la technique Elisa, chez 14 sujets parmi les 39 chevaux suspects d'être atteints par cette infection (35,9 p. 100).

Ce travail a montré une différence significative entre la prévalence de l'infection chez les chevaux suspects et celle des chevaux sains considérés comme témoins. C'est ainsi que la prévalence de l'infection à Rhodococcus equi serait liée à l'intervention de souches virulentes et aussi à la présence d'un nombre important de bactéries dans l'environnement. En effet, la mise en évidence d'un système VAP A a permis de distinguer les souches pathogènes des souches apathogènes de Rhodococcus equi. Ce système de virulence VAP A a été de ce fait utilisé dans le présent travail dans le cadre d'une technique de diagnostic sérologique Elisa mise au point par le Laboratoire d'études et de recherches en pathologie équine. Cette technique a permis un diagnostic précoce de l'infection, mais elle reste toutefois inaccessible pour une utilisation routinière avec parfois de fausses réponses positives.

Cette étude devrait être complétée par des travaux visant à explorer un nombre beaucoup plus important de chevaux et à établir une éventuelle corrélation entre le nombre de bactéries dans l'environnement et l'impact de la maladie dans les élevages équins.

L'isolement de la souche serait aussi intéressant à faire surtout à partir du liquide broncho-alvéolaire ou de cadavres d'animaux dont l'autopsie confirme qu'ils sont morts de cette infection.

Ces connaissances réunies permettraient d'instaurer une prévention médicale et sanitaire efficace de l'infection à Rhodococcus equi chez les poulains et les chevaux en Tunisie.

\section{BIBLIOGRAPHIE}

1. BARTON M.D., 1991. The ecology and epidemiology of Rhodococcus equi. In: Proc. American Association of Equine Practitioners. Equine infect. Dis., 1: 77-81.

2. COLLOBERT C., 1997. Les arthrites à Rhodococcus equi chez le poulain. Prat. vét. équine, 29 : 245-246.

3. COLLOBERT C., DENOIX I.M., 1990. L'infection à Corynebacterium equi chez le poulain. Prat. vét. équine, $3:$ 41-47.

\section{Summary}

Chabchoub A., Taouji S., Landolsi F., Collobert C., Chtioui Z., Messadi L., Ezzaouia M., Ghorbel A. Contribution to the Study of Rhodococcus equi Infections in Horses in the Northeast of Tunisia

The authors searched by ELISA for antibodies directed against virulent protein VAP A of Rhodococcus equi in 39 horses that displayed Rhodococcus infection-like symptoms, and in 57 healthy foals used as control. Among sick horses and control foals, 35.9 and $17.5 \%$ were positive, respectively. No significant differences were found based on sex or breed. But significant differences were found between the various age groups of the studied horses.

Key words: Equidae - Horse - Rhodococcus equi - ELISA Tunisia.
4. COLLOBERT C., FOURSIN M., FABRY A.C., TARIEL G., LAMIDEY C., FOUCHER N., FORTIER G., MOUSSU C., 1998. Infection à Rhodococcus equi chez le poulain à l'autopsie : importance des localisations ostéoarticulaires et musculaires. Prat. vét. équine, 30 : 27-34.

5. FALCON J., SMITH B.P., O'BRIEN T.R., CARLSON G.P., BIBERSTEIN E., 1985. Clinical and radiographic finding in Corynebacterium equi pneumonia of foals. J. Am. vet. Med. Assoc., 186: 593-599.

6. HIGUCHI T., HASHIKURA S., GOJO C., INUI T., SATOH S., YOSHIDA M., ISHIYAMA T., YAMADA H., TAKAI S., 1997. Clinical evaluation of the serodiagnostic value of enzyme-linked immunosorbent assay for Rhodococcus equi infection in foals. Equine vet. J., 29: 274-278.

7. HIGUCHI T., TAHARAGUCHI S., HASHIKURA S., HAJIWARA S., KOJO C., SATOH S., YOSHIDA M., TAKAI S., 1998. Physical and serologic examinations of foals at 30 and 45 days of age for early diagnosis of Rhodococcus equi infection in endemically infected farms. J. Am. vet. Med. Assoc., 212: 976-981.

8. HOFMANN A., VIEL L., PRESCOTT J.F., ROSENDAL S., THORSEN J., 1993. Association of microbiologic flora with clinical, endoscopic, and pulmonary cytologic findings in foals with distal respiratory tract infection. Am. J. vet. Res., 54: 1615-1622.

9. HONDALUS M.K., 1997. Pathogenesis and virulence of Rhodococcus equi. Vet. Microbiol., 56: 257-268.

10. KNOTTENBELT D.C., 1993. Rhodococcus equi infection in foals: A report of an outbreak on a thoroughbred stud in Zimbabwe. Vet. Rec., 23: 79-85.

11. MARTENS R.J., FISHE R.A., RENSHAW H.W., 1982. Experimental subacute foal pneumonia induced by aerosol administration of Corynebacterium equi. Equine vet. J., 14: 111-116.

12. PRESCOTT J.F., FERNANDEZ A.S., NICHOLSON V.M., PATTERSON M.C., YAGER J.A., VIEL L., PERKINS G., 1996 Use of virulence associated protein-based enzyme-linked immunosorbent assay for Rhodococcus equi serology in horses. Equine vet. J., 28: 233-349.

13. PRESCOTT J.F., SWEENY C.R., 1985. Treatment of Corynebacterium equi pneumonia of foals: A review. J. Am. vet. Med. Assoc., 187: 725-728.

14. PRONOST S., FORTIER G., LEGENDRE M.F., OGAWA K., SASAKI Y., COLLOBERT C., TAOUGI S., TAKAI S., 1998. Rhodococcose équine: nouvelles approches et bilan de trois années d'études au laboratoire. Prat. vét. équine, 30 : 57-65.

15. ROBERTS M.C., HODSON D.R., WELLY W.R., 1980. Corynebacterium equi: infection in adult horse. Aust. vet. J., 56: 96-97.

16. SCOTT M.A., GRAHAM B.S., VERRALL R., DIXON R., SCHAFFNER W., THAM K.T., 1995. Rhodococcus equi - An increasingly recognized opportunistic pathogen. Report of 12 cases and review of 65 cases in the literature. Am. J. clin. Pathol., 103: 56-59.

17. TAKAI S., KAWAZU S., TSUBAKI S., 1985. Enzyme-linked immunosorbent assay for diagnosis of Corynebacterium (Rhodococcus) equi infection in foals. Am. J. vet. Res., 46: 2166-2170.

Reçu le 30.03.2001, accepté le 05.03.2002

\section{Resumen}

Chabchoub A., Taouji S., Landolsi F., Collobert C., Chtioui Z., Messadi L., Ezzaouia M., Ghorbel A. Contribución al estudio de la infección por Rhodococcus equi en los equinos en el nordeste de Túnez

Los autores buscaron, mediante la técnica de ELISA, la presencia de anticuerpos anti proteína de virulencia VAP A de Rhodococcus equi, en 39 caballos que presentaron síntomas evocadores de rodococosis y 57 potros sanos considerados como testigos. Entre los caballos enfermos, 35,9\% fueron positivos contra $17,5 \%$ únicamente en los testigos. No se encontró ninguna diferencia en función del sexo ni de la raza. Sin embargo, los resultados serológicos muestran diferencias estadísticamente significativas entre los grupos de edad de los caballos estudiados.

Palabras clave: Equidae - Caballo - Rhodococcus equi ELISA - Túnez. 